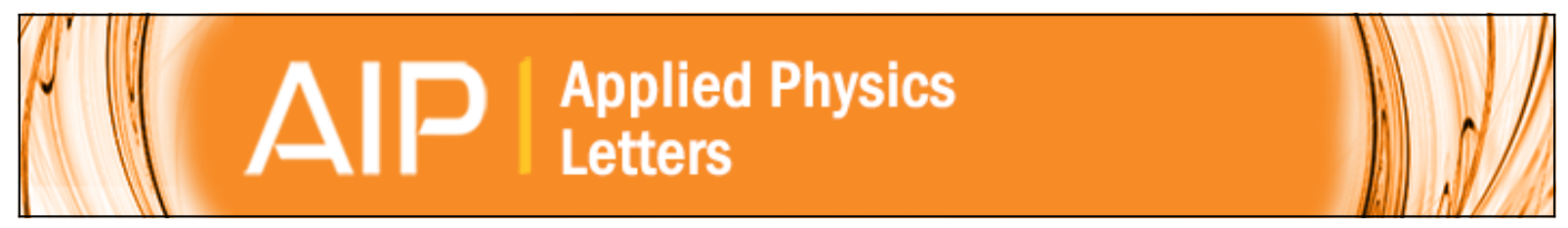

Tuning nonlinearity, dynamic range, and frequency of nanomechanical resonators

I. Kozinsky, H. W. Ch. Postma, I. Bargatin, and M. L. Roukes

Citation: Applied Physics Letters 88, 253101 (2006); doi: 10.1063/1.2209211

View online: http://dx.doi.org/10.1063/1.2209211

View Table of Contents: http://scitation.aip.org/content/aip/journal/apl/88/25?ver=pdfcov

Published by the AIP Publishing

Articles you may be interested in

Electrostatically actuated silicon-based nanomechanical switch at room temperature

Appl. Phys. Lett. 93, 033515 (2008); 10.1063/1.2964196

Two-dimensional array of coupled nanomechanical resonators

Appl. Phys. Lett. 88, 143504 (2006); 10.1063/1.2190448

Silicon nanomechanical resonators with a double-triangle cross section leading to an enhanced mass sensitivity Appl. Phys. Lett. 88, 063112 (2006); 10.1063/1.2172236

Mechanical gating of coupled nanoelectromechanical resonators operating at radio frequency

Appl. Phys. Lett. 82, 352 (2003); 10.1063/1.1536032

Mechanical mixing in nonlinear nanomechanical resonators

Appl. Phys. Lett. 77, 3102 (2000); 10.1063/1.1324721

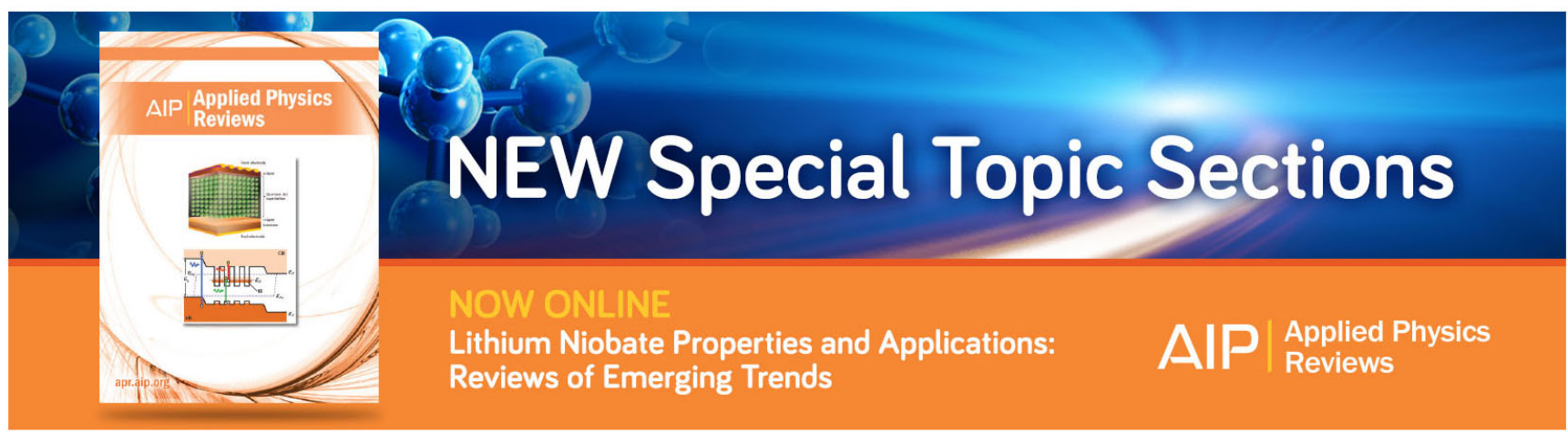




\title{
Tuning nonlinearity, dynamic range, and frequency of nanomechanical resonators
}

\author{
I. Kozinsky, H. W. Ch. Postma, I. Bargatin, and M. L. Roukes ${ }^{a)}$ \\ Condensed Matter Physics 114-36, California Institute of Technology, Pasadena, California 91125
}

(Received 20 December 2005; accepted 19 April 2006; published online 19 June 2006)

\begin{abstract}
We explore an electrostatic mechanism for tuning the nonlinearity of nanomechanical resonators and increasing their dynamic range for sensor applications. We also demonstrate tuning the resonant frequency of resonators both upward and downward. A theoretical model is developed that qualitatively explains the experimental results and serves as a simple guide for design of tunable nanomechanical devices. (C) 2006 American Institute of Physics. [DOI: 10.1063/1.2209211]
\end{abstract}

In the past few years, nanoelectromechanical systems (NEMS) have been used for a variety of sensing, imaging, and detection applications. Among these are devices for zeptogram scale mass sensing, ${ }^{1}$ single electron spin detection, ${ }^{2}$ and RF communications. ${ }^{3}$ All of these applications require a large dynamic range, over which the device responds linearly to the stimulus, as well as operation at desired and, preferably, adjustable frequencies. However, we have recently shown ${ }^{4}$ that as nanomechanical beam resonators become smaller, their dynamic range decreases, making them less useful as linear sensors. The ability to tune nonlinearity, and consequently, dynamic range, allows us to overcome this limitation, which can be especially pronounced in NEMS based on high aspect ratio structures such as nanotubes and nanowires. In addition, a controllable method of tuning provides a way to adjust resonant frequency both for control applications such as phase locking and to null inevitable, fabrication-induced device variations.

In this letter we demonstrate an electrostatic mechanism for tuning the nonlinearity in nanomechanical resonators. As a direct consequence, it becomes possible to increase their dynamic range. In addition, we demonstrate an ability to tune the resonant frequency of resonators both upward and downward and evaluate the electromechanical dissipation associated with different regimes of frequency tuning. The results are described by a model that qualitatively agrees with the experiment and can serve as a simple guide for design of tunable nanomechanical devices.

The inset to Fig. 1 shows the device used for the tuning measurements: a suspended metallized $\mathrm{SiC}$ beam ${ }^{5} 150 \mathrm{~nm}$ wide, $100 \mathrm{~nm}$ thick, and $15 \mu \mathrm{m}$ long is positioned $400 \mathrm{~nm}$ away from a gate electrode, to which a dc bias can be applied. Built-in strain in the multilayered structure yields an intrinsic tension within the beam, raising the resonant frequency from the calculated $4.7 \mathrm{MHz}$ to the observed $8.78 \mathrm{MHz}$. From the model below, we calculate this residual tension to be $5.3 \mu \mathrm{N}$. The mechanical resonant characteristics of the beam were measured using the magnetomotive driving and detection technique ${ }^{6}$ in a $7 \mathrm{~T}$ magnetic field. The beam's resonant frequency was measured to be $8.78 \mathrm{MHz}$ for the in-plane mode and $7.60 \mathrm{MHz}$ for the out-of-plane mode.

\footnotetext{
a) Author to whom correspondence should be addressed; electronic mail: roukes@caltech.edu
}

Figure 1 shows a typical resonant response of the beam, where the vibration is in the plane of the gate and the gate is grounded. As the drive amplitude is increased, the response becomes nonlinear. In such doubly clamped beams, the origin of nonlinearity is the additional tension that appears when the beam vibrates with sufficiently large amplitude. The nonlinearity can be characterized by the critical response amplitude $a_{c}$, the point where the resonance curve develops infinite slope (onset of hysteresis). ${ }^{4,7}$ We determine $a_{c}$ from the "backbone" curve (shown as a solid red line in Fig. 1) connecting peaks of resonant curves for different drives when the frequency is swept upward.

The electrostatic tuning setup, shown in the inset of Fig. 1 , allows us to tune both nonlinearity and the resonant frequency of the beam. Figure 2 shows that critical amplitude increases with dc gate bias. In other words, as the gate voltage is increased, the onset of nonlinearity occurs at higher drives. This translates into a less nonlinear behavior and, consequently, into the increase in the dynamic range of the device. Figure 2 shows the dynamic range growth with the gate voltage, calculated from the measured critical amplitude values and the experimental noise floor of $1 \mathrm{nV} / \sqrt{\mathrm{Hz}}$. In general, the ultimate noise floor limit is set by thermomechanical noise and quantum fluctuations.

The physical reason for the observed nonlinearity tuning is the appearance of even-ordered nonlinear terms in the equation of motion. The effective nonlinearity of the system is $\alpha=\alpha_{3}-\left(10 / 9 \omega_{0}\right) \alpha_{2}^{2}$, where $\alpha_{3}$ and $\alpha_{2}$ are cubic and quadratic nonlinear coefficients, respectively. ${ }^{7}$ The cubic nonlinearity is primarily due to the elastic properties of the beam.

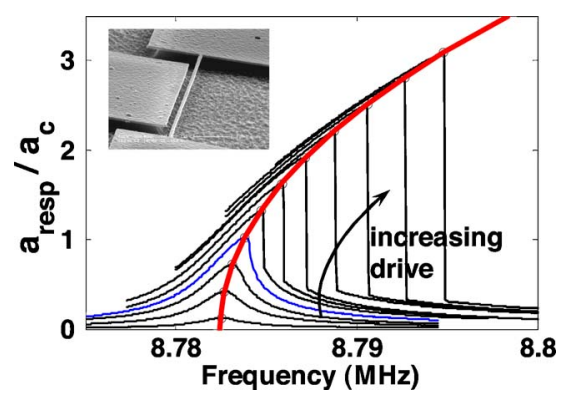

FIG. 1. (Color online) Family of resonant response curves of the suspended $\mathrm{SiC}$ doubly clamped beam to a progressively increased drive. The solid red line shows a backbone curve used to characterize the onset of nonlinearity: $f_{\text {peak }}=f_{0}\left(1+1 / \sqrt{3} Q\left(a_{\text {peak }} / a_{c}\right)^{2}\right)$. SEM image of the device is shown in the inset. 


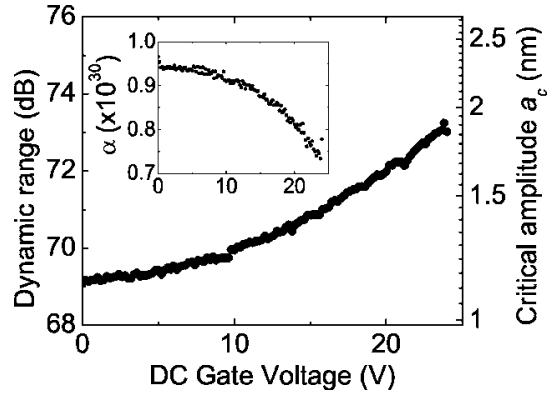

FIG. 2. Variation of dynamic range and critical amplitude with the gate voltage. The inset shows the observed decrease in effective nonlinearity $\alpha$ with the gate voltage.

The quadratic nonlinearity arises when the symmetry of the beam's displacement from its central axis is broken by the capacitive attraction to the gate. dc bias voltage on the gate increases quadratic nonlinearity in the beam, which decreases the overall nonlinearity and results in larger values for the critical amplitude. The observed decrease in the effective nonlinearity $\alpha$ with the dc gate voltage is shown in the inset of Fig. 2. The possibility of such a nonlinearity tuning mechanism has been suggested by Younis and Nayfeh, ${ }^{8}$ but we are not aware of any experimental realization of nonlinearity tuning in mechanical resonators.

Using the same setup, we can also tune the resonant frequency of the beam. Figure 3 illustrates the results obtained by varying the dc bias applied to the gate electrode. When the out-of-plane mode of vibration is excited, we observe an increase in the resonant frequency of the beam with gate voltage [Figs. 3(a) and 3(b)]. As the applied gate voltage pulls the beam toward the gate, the resonant frequency increases, similar to the increase in pitch produced by stretching a guitar string. Since the quality factor remains constant throughout the increase in the dc gate voltage [inset of Fig. 3(b)], this mechanism is well suited for applications where a change in frequency is the only desired effect.

For the in-plane mode, we observe the decrease in the resonant frequency [Figs. 3(c) and 3(d)]. The beam is electrostatically attracted to the gate and this makes its spring constant smaller. This tuning is accompanied by increased dissipation [inset of Fig. 3(d)] as the modulated capacitance between the beam and the gate gives rise to dissipative current flow.

Various reversible frequency-tuning mechanisms have been explored in the past. These include scanning tunneling microscope (STM)-aided resonator length adjustment, ${ }^{9}$ stiffness variation with thermal stress, ${ }^{10}$ and electrostatic tuning. ${ }^{11,12}$ The frequency has been observed to tune either up $^{12}$ or down, ${ }^{11}$ and the theoretical models built to explain the data took into account a single tuning mechanism, either elastic or capacitive, that seemed dominant in that particular experiment.

In our experiment, we observe both softening and hardening types of frequency tuning in the same device. Here we present a model that reconciles the existence of these two types as it takes into account elastic and capacitive frequency tuning as well as the beam nonlinearity. The model reliably predicts the decrease in nonlinearity and increase in dynamic range as well as the two frequency tuning mechanisms that we observe.
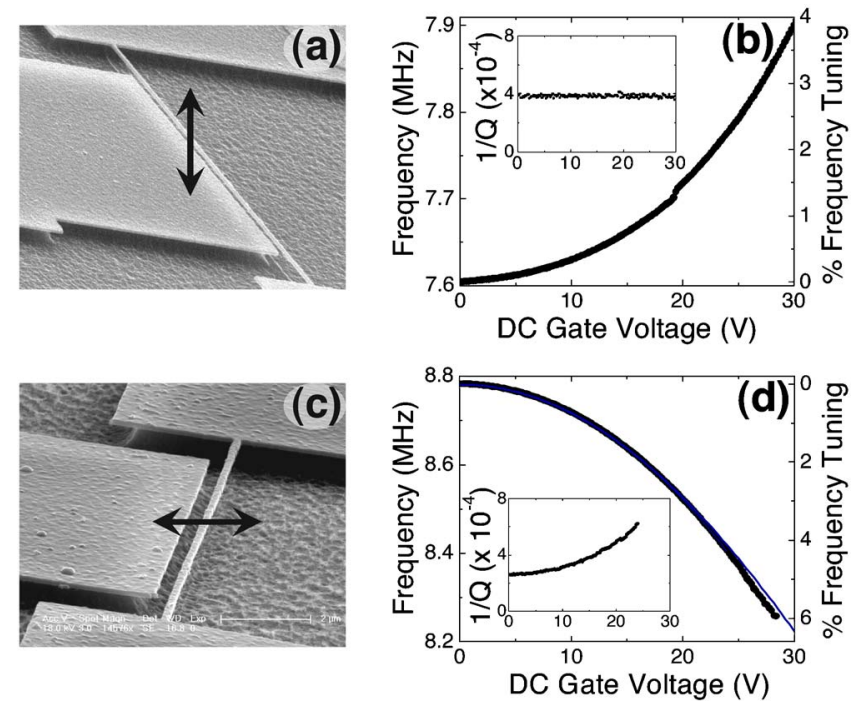

FIG. 3. (Color online) Elastic tuning of frequency upward (b) for the beam's vibration out of plane with the gate (a). Capacitive tuning of frequency downward (d) for vibration in plane of the gate (c). The blue curve in (d) is the prediction of the theoretical model for the capacitive frequency tuning.

Our device is a thin beam that is displaced by dc bias voltage on the gate and driven by a small ac voltage. We can write the beam's total displacement $u(x, t)$ as a sum of a static dc displacement $z_{s}(x)$ and a time-varying ac displacement $z(x, t): u(x, t)=z_{s}(x)+z(x, t)$.

The combination of the electrostatic force, attracting the beam towards the gate, and the elastic restoring force, trying to pull the beam back to its undeformed state, gives us the equation of motion for the beam as follows:

$$
E I u_{x x x x}-\left[T_{0}+T\left(u_{x}\right)\right] u_{x x}+\rho S u_{t t}=\frac{1}{2} c_{z}[z(x, t)] V^{2},
$$

with the boundary condition at the two clamped ends. $S$ is the beam's cross-sectional area, $E$ is Young's modulus, $\rho$ is the beam density, and $I$ is the moment of inertia about the longitudinal axis of the beam. The total tension term in brackets is a sum of residual tension $T_{0}$ and bending-induced tension: $T\left(u_{x}\right)=(E S / 2 L) \int_{0}^{L} u_{x}^{2} d x$, where $L$ is the beam length. The capacitance per unit length $c[z(x, t)]$ is calculated using an approximation of an infinite wire near the semi-infinite plane of the gate ${ }^{13}$ and expanded to the fourth order in $z(x, t) / R$. The capacitance expansion coefficients $K_{i}$ are given by the geometry of the system and the dc displacement of the beam.

To find the frequency and nonlinear coefficients in the equation for the time-varying displacement, we approximate the beam shape as $z(x, t)=z_{1}(t) \sqrt{2 / 3}[1-\cos (2 \pi x / L)] .^{4,14} \mathrm{~A}$ similar expansion of static deflection, $z_{s}(x, t)=A_{\mathrm{dc}} \sqrt{2 / 3}[1$ $-\cos (2 \pi x / L)]$, is used in the static equilibrium equation to solve for the static deflection amplitude $A_{\mathrm{dc}}$. The Galerkin discretization procedure ${ }^{7}$ gives the time-variant part of the equation of motion:

$$
\ddot{z}_{1}(t)+\omega_{0}^{2} z_{1}(t)+\alpha_{2} z_{1}^{2}(t)+\alpha_{3} z_{1}^{3}(t)=0,
$$

where

$$
\omega_{0}^{2}=\left[\frac{E I}{3 \rho S}+\frac{E A_{\mathrm{dc}}^{2}}{6 \rho}\right]\left(\frac{2 \pi}{L}\right)^{4}+\frac{T_{0}}{3 \rho S}\left(\frac{2 \pi}{L}\right)^{2} \frac{K_{2} V^{2}}{\rho S}
$$




$$
\begin{aligned}
& \alpha_{3}=\frac{E}{18 \rho}\left(\frac{2 \pi}{L}\right)^{4}-\frac{35}{9} \frac{K_{4} V^{2}}{\rho S}(4), \\
& \alpha_{2}=\frac{E A_{\mathrm{dc}}}{6 \rho}\left(\frac{2 \pi}{L}\right)^{4}-\frac{5}{2} \sqrt{\frac{2}{3}} \frac{K_{3} V^{2}}{\rho S} .
\end{aligned}
$$

The resulting equation of motion (2) predicts the observed tuning behavior. The overall nonlinearity is reduced because the quadratic nonlinearity (3) grows with static dc deflection due to the voltage applied to the gate and partially cancels out the cubic nonlinearity: $\alpha=\alpha_{3}-\left[10 /\left(9 \omega_{0}\right) \alpha_{2}^{2}\right]$. $^{7}$ The decrease in $\alpha_{3}$ itself with the increased dc voltage is a higher-order effect and is negligible compared to the increase in $\alpha_{2}$.

Compared to the experimental data, the model predicts a sharper increase in $a_{c}$ at higher dc voltages. In the experiment, the increase in critical amplitude is probably limited by higher-order and nontension nonlinearities, e.g., material and inertial nonlinearities, ${ }^{15}$ that become significant when the induced quadratic nonlinearity cancels the cubic nonlinearity.

The different frequency tuning behaviors are also explained by Eq. (3). The frequency increases in the out-ofplane vibration mode because increasing the gate voltage only stretches the beam (increasing static displacement $A_{\mathrm{dc}}$ ), but does not interact with it electrostatically. For the in-plane vibration mode, there is both stretching (increase in $A_{\mathrm{dc}}$ ) and electrostatic interaction (described by the $K_{2} V^{2}$ term). Electrostatic attraction to the gate has a softening effect on the beam for low gate voltages before the hardening due to stretching overcomes it. If the mass loading by a metal layer on the $\mathrm{SiC}$ beam is taken into account, the theoretical expression for resonant frequency (3) can be fitted to the data with excellent agreement as shown in Fig. 3(d).

The ultimate limit to electrostatic tuning in these devices depends on the pull-in voltage when the beam gets close enough to the gate to irreversibly snap in. ${ }^{16}$ The pull-in voltages for the geometry that we describe here were observed to range from 29 to $42 \mathrm{~V}$.

A device that has an additional gate electrode below or above the beam should allow tuning of frequency both up and down for the same mode of the resonator if the two electrodes can be biased independently. Such a device could also be used to keep the frequency of the beam constant while tuning its dynamic range.

An interesting application of frequency tuning is the ability to controllably couple the in-plane and out-of-plane modes as the frequencies of the two modes are tuned closer to each other. For the geometry that we describe here, the avoided mode crossing was observed at $33.5 \mathrm{~V}$ with the frequency gap of $0.1 \mathrm{MHz}$.

In this letter, we present an experimental observation of electrostatic tuning of the onset of nonlinearity and, consequently, of the tuning of dynamic range of nanomechanical resonators. We also demonstrate the ability to tune resonant frequencies of our devices upward and downward. These mechanisms and their analysis provided here are helpful for any applications requiring adjustable frequency or dynamic range. Among these are frequency adjustment to overcome fabrication inaccuracy and embedding a tunable NEMS resonator in a feedback loop to allow for real-time frequency stabilization. Nonlinearity tuning will allow applications of small and sensitive devices (such as single-wall nanotubes) as linear sensors. The proposed theoretical model can be used to experiment with different geometries to optimize frequency or nonlinearity tuning and to increase the pull-in threshold for specific applications.

The authors acknowledge support from DARPA/MTO (SPAWAR) under Grant No. N66001-02-1-8914. The authors thank C. A. Zorman and M. Mehregany for providing SiC wafers, and are grateful to X. L. Feng, O. Kogan, M. C. Cross, and A. Husain for useful discussions.

${ }^{1}$ Y. T. Yang, C. Callegari, X. L. Feng, K. L. Ekinci, and M. L. Roukes, Nano Lett. 6, 583 (2006).

${ }^{2}$ D. Rugar, R. Budakian, H. Mamin, and B. Chui, Nature (London) 430, 329 (2004).

${ }^{3}$ C.-C. Nguyen, IEEE Trans. Microwave Theory Tech. 47, 1486 (1999).

${ }^{4}$ H. W. C. Postma, I. Kozinsky, A. Husain, and M. L. Roukes, Appl. Phys. Lett. 86, 223105 (2005)

${ }^{5}$ Y. T. Yang, K. L. Ekinci, X. M. H. Huang, L. M. Schiavone, M. L. Roukes, C. A. Zorman, and M. Mehregany, Appl. Phys. Lett. 78, 162 (2001).

${ }^{6}$ A. Cleland and M. Roukes, Sens. Actuators, A 72, 256 (1998).

${ }^{7}$ A. H. Nayfeh and D. T. Mook, Nonlinear Oscillations, 1st ed. (Wiley, New York, 1979),

${ }^{8}$ M. Younis and A. H. Nayfeh, Nonlinear Dyn. 31, 91 (2003).

${ }^{9}$ M. Zalalutdinov, B. Ilic, D. Czaplewski, A. Zehnder, H. G. Craighead, and J. M. Parpia, Appl. Phys. Lett. 77, 3287 (2000).

${ }^{10}$ R. Syms, J. Microelectromech. Syst. 7, 164 (1998).

${ }^{11}$ K. Schwab, Appl. Phys. Lett. 80, 1276 (2002).

${ }^{12}$ V. Sazonova, Y. Yaish, H. Uestuenel, D. Roundy, T. A. Arias, and P. L. McEuen, Nature (London) 431, 284 (2004).

${ }^{13} \mathrm{~B}$. Bleaney and B. Bleaney, Electricity and Magnetism (Oxford University Press, Oxford, 1989).

${ }^{14}$ J. G. Eisley, J. Appl. Math Phys. 15, 167 (1964).

${ }^{15}$ M. R. M. Crespo da Silva and C. C. Glynn, J. Struct. Mech. 6, 437 (1978).

${ }^{16}$ E. Buks and M. L. Roukes, Europhys. Lett. 54, 220 (2001). 